\title{
Therapeutic Drug Monitoring Simulator for Antibiotic Dosage for Methicillin-Resistant Staphylococcus aureus Sepsis in a Patient with Primary Immunodeficiency on Peritoneal Dialysis
}

\author{
Hidehiko Narazaki ${ }^{1}$, Yusuke Terada ${ }^{1}$, Kiyohiko Kaizu $^{1}$, \\ Toyo Jitsukawa ${ }^{2,3}$, Yasuhiko Ito ${ }^{1}$ and Takeshi Asano ${ }^{1}$ \\ ${ }^{1}$ Department of Pediatrics, Nippon Medical School, Tokyo, Japan \\ ${ }^{2}$ Department of Pharmacy, Nippon Medical School, Chiba Hokuso Hospital, Chiba, Japan \\ ${ }^{3}$ Division of Infection Control and Prevention, Nippon Medical School, Chiba Hokusoh Hospital, Chiba, Japan
}

\begin{abstract}
Bacterial infections often cause fatal systemic infections in patients with primary immunodeficiency. To prevent unfortunate results, the selection, dose, and dosage of antibiotics are extremely important. Here, we report a case of Wiskott-Aldrich syndrome in a patient undergoing peritoneal dialysis because of chronic renal failure in whom methicillin-resistant Staphylococcus aureus sepsis developed. Because of the primary disease and complications, teicoplanin was the only chosen anti-S. aureus drug to prevent side effects. We used parameter estimation and dosage adjustment from a therapeutic drug monitoring simulation software program to overcome the challenges with teicoplanin treatment.
\end{abstract}

(J Nippon Med Sch 2017; 84: 177-182)

Key words: drug monitoring, primary immunodeficiency disorders, methicillin-resistant Staphylococcus aureus, sepsis, peritoneal dialysis

\section{Introduction}

During the long-term survival course of patients with primary immunodeficiency, several complications often occur, particularly severe multidrug- resistant bacterial infections, which can affect prognosis. Thus, the selection of the correct antibiotic and optimal plasma drug concentrations is important, especially for multidrug-resistant organisms, such as methicillin-resistant Staphylococcus aureus (MRSA).

The area under the blood concentration-time curve/ minimum inhibitory concentration is extremely important for the antimicrobial activity of glycopeptides, and the trough value is also an important consideration to prevent side effects. However, drug metabolism varies among patients; thus, determining the proper dose to maintain adequate plasma drug concentrations is difficult. Therefore, therapeutic drug monitoring (TDM) is extremely important.

Wiskott-Aldrich syndrome is a congenital disease that is characterized by the triad of thrombocytopenia, refrac- tory eczema, and immunodeficiency ${ }^{1}$. However, WiskottAldrich syndrome also includes autoimmune manifestations and tumors.

In this paper, we report a case of MRSA sepsis in a patient with Wiskott-Aldrich syndrome who underwent peritoneal dialysis because of chronic renal failure.

\section{Case}

At 7 years of age, the patient presented with thrombocytopenia, refractory eczema, elevated serum immunoglobulin (Ig)E level, decreased serum IgM level, and recurrent infections. Wiskott-Aldrich syndrome was diagnosed with gene analysis (guanine deletion at exon 8 of the Wiskott-Aldrich syndrome gene [WAS]). At 17 years of age, acute renal failure developed because of Klebsiella spp. sepsis. The general condition improved after the patient was admitted to our hospital, but hematuria and proteinuria persisted. Although we considered kidney biopsy, we did not perform it because the platelet count was extremely low (approximately 10,000/ $\mathrm{LL}$ ). On the

Correspondence to Hidehiko Narazaki, MD, PhD, Department of Pediatrics, Nippon Medical School, 1-1-5 Sendagi, Bunkyoku, Tokyo 113-8603, Japan

E-mail: nara@nms.ac.jp

Journal Website (http://www2.nms.ac.jp/jnms/) 
Table 1 Laboratory data on the day of admission

\begin{tabular}{lclc}
\hline White blood cell & $10,120 / \mathrm{mL}$ & Alanine aminotransferase & $15 \mathrm{U} / \mathrm{L}$ \\
Stab cell & $2 \%$ & Lactate dehydrogenase & $469 \mathrm{U} / \mathrm{L}$ \\
Segmented cell & $83 \%$ & Creatine kinase & $243 \mathrm{U} / \mathrm{L}$ \\
Lymphocyte & $4 \%$ & C-reactive protein & $16.23 \mathrm{mg} / \mathrm{dL}$ \\
Monocyte & $3 \%$ & PT-INR & 1.16 \\
Eosinophil granulocyte & $8 \%$ & APTT & $36.0 \mathrm{sec}$ \\
Red blood cell & $2.2 \times 10^{6} / \mathrm{mL}$ & TT-INR & 0.93 \\
Hemoglobin & $6.7 \mathrm{~g} / \mathrm{dL}$ & Hepaplastin test & $98.3 \%$ \\
Hematocrit & $21.1 \%$ & Fibrinogen & $399 \mathrm{mg} / \mathrm{dL}$ \\
Platelets & $8,000 / \mathrm{mL}$ & Fibrinogen degradation products & $19.6 \mathrm{mg} / \mathrm{mL}$ \\
Total protein & $5.9 \mathrm{~g} / \mathrm{dL}$ & Sodium & $139 \mathrm{mEq} / \mathrm{L}$ \\
\hline Albumin & $3.0 \mathrm{~g} / \mathrm{dL}$ & Potassium & $7.2 \mathrm{mEq} / \mathrm{L}$ \\
\hline Blood urea nitrogen & $71.8 \mathrm{mg} / \mathrm{dL}$ & Chloride & $103 \mathrm{mEq} / \mathrm{L}$ \\
\hline Creatinine & $13.63 \mathrm{mg} / \mathrm{dL}$ & Calcium & $6.2 \mathrm{mg} / \mathrm{dL}$ \\
\hline Aspartate aminotransferase & $19 \mathrm{U} / \mathrm{L}$ & & \\
\hline PT-INR, international normalized ratio of prothrombin time; APTT, activated partial thromboplastin time; TT- \\
INR, international normalized ratio of thrombin time
\end{tabular}

basis of clinical symptoms and previous case reports ${ }^{2}$, we suspected an autoimmune kidney disease, such as $\operatorname{Ig} \mathrm{A}$ nephropathy.

Prednisolone administration was started. Renal function improved, but the blood pressure gradually increased. Unfortunately, the prednisolone dosage had to be reduced. As a result, renal function gradually decreased. Chronic renal failure eventually developed, and peritoneal dialysis was started when the patient was 19 years old. At 21 years of age, he was admitted to our hospital because of continuous bleeding from impetigo of the right thigh. At the same time, the serum potassium level was elevated because of peritoneal dialysis had failed (Table 1). Levofloxacin was administered (500 mg on the first day, followed by $250 \mathrm{mg}$ every other day), according to the Johns Hopkins Antibiotic Guide ${ }^{3}$.

Maintaining hemostasis of the thigh wound was difficult, and platelet transfusion was required. The hyperkalemia soon decreased with the administration of furosemide and sodium polystyrene sulfonate. On hospital day 8 , the patient had a high fever, headache, and general fatigue. We suspected infection with a drug-resistant pathogen because levofloxacin had already been administered. Meropenem and clindamycin were administered for empiric therapy after 2 blood samples for blood culture were collected. In both samples collected the next day MRSA was detected.

Treatment with teicoplanin was started on hospital day 9. The antibiotics TDM guideline from the Japanese Soci- ety of Chemotherapy ${ }^{4}$ recommends that $400 \mathrm{mg}(6 \mathrm{mg}$ / $\mathrm{kg}$ ) of teicoplanin should be administered twice daily for the first 3 days and that the trough concentration of teicoplanin should be measured on day 4 regardless of renal function. Treatment with teicoplanin was started at a dose of $400 \mathrm{mg}$ twice daily, but the teicoplanin trough concentration reached $30.0 \mu \mathrm{g} / \mathrm{mL}$ before the fifth dose was administered. We decided to re-evaluate the dosage. We developed 3 patterns and estimated the teicoplanin trough concentrations of each pattern using parameter estimation and dosage adjustment (PEDA) ${ }^{5}$, a TDM simulation software program, on the basis of renal function (Fig. 1).

On the basis of the simulation, $300 \mathrm{mg}$ of teicoplanin was administered on day 4 before we confirmed the teicoplanin trough concentration. Unexpectedly, the teicoplanin concentration was $29.1 \mu \mathrm{g} / \mathrm{mL}$ teicoplanin was administered on day 4 before we confirmed the reevaluated his renal function and recalculated the dosage. We estimated that the teicoplanin concentration was $19.04 \mu \mathrm{g} / \mathrm{mL}$ (Fig. 2A). However, because the teicoplanin concentration decreased more slowly than expected, recalculation was necessary. As a result, we planned to administer $200 \mathrm{mg}$ of teicoplanin daily until day 17 (Fig. 2 B). We continued to evaluate the teicoplanin concentration and the efficacy of peritoneal dialysis and to titrate the dosage per the recalculation results from PEDA and the patient's condition (Fig. 2C, D). Finally, we administered teicoplanin at irregular intervals for 6 weeks and 
A.

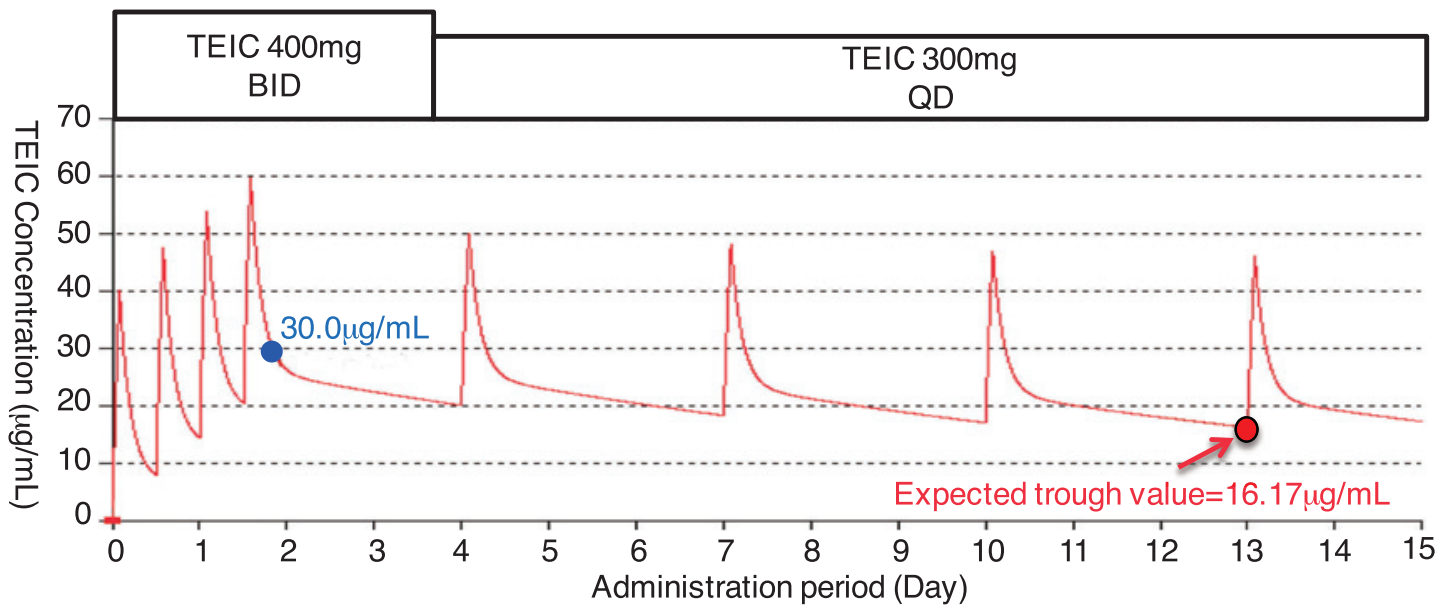

B.

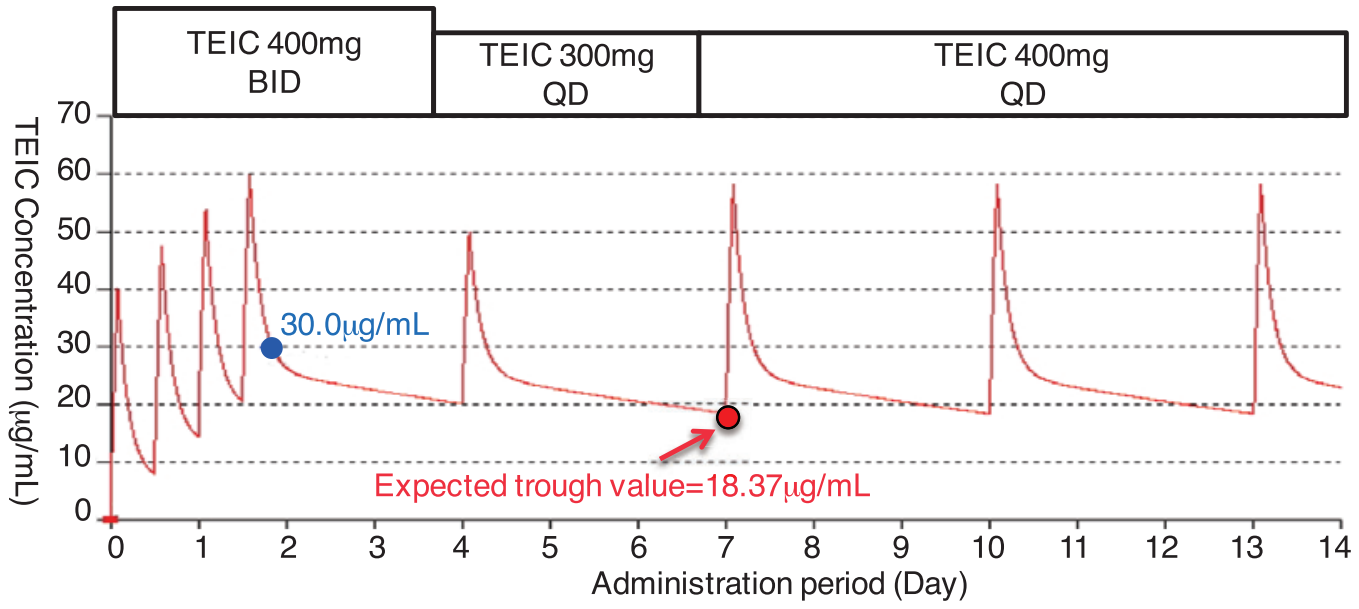

C.

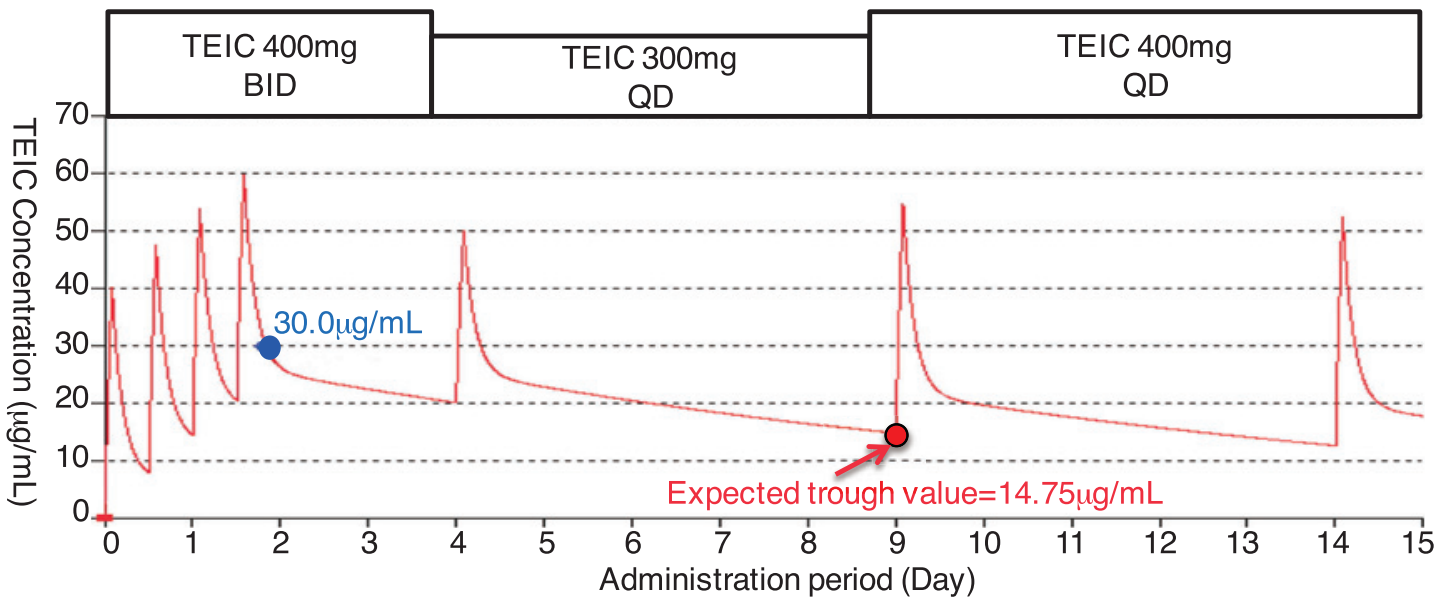

Fig. 1 We formulated 3 treatment patterns and estimated the teicoplanin trough concentrations with each pattern using a therapeutic drug monitoring simulation software program and the patient's renal function.

A: Model showing administration of $300 \mathrm{mg}$ twice daily, every 3 days starting on day 4. B: Model showing administration of $300 \mathrm{mg}$ twice daily on day 4 and $400 \mathrm{mg}$ twice daily, every 3 days starting on day 7. C: Model showing administration of 300 mg twice daily on day 4 and $400 \mathrm{mg}$ twice daily, every 5 days starting on day 7 . The blue dots show the absolute teicoplanin blood concentrations. The red line shows the calculated teicoplanin blood concentration. The red dot shows the expected teicoplanin trough concentrations. 
A.

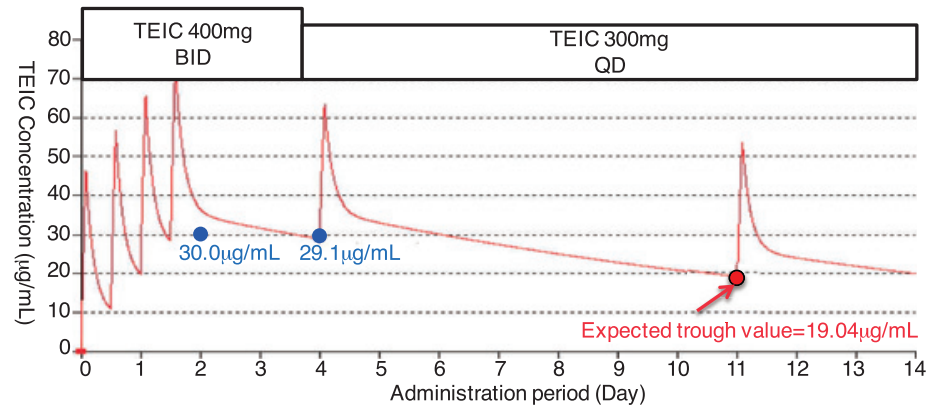

B.

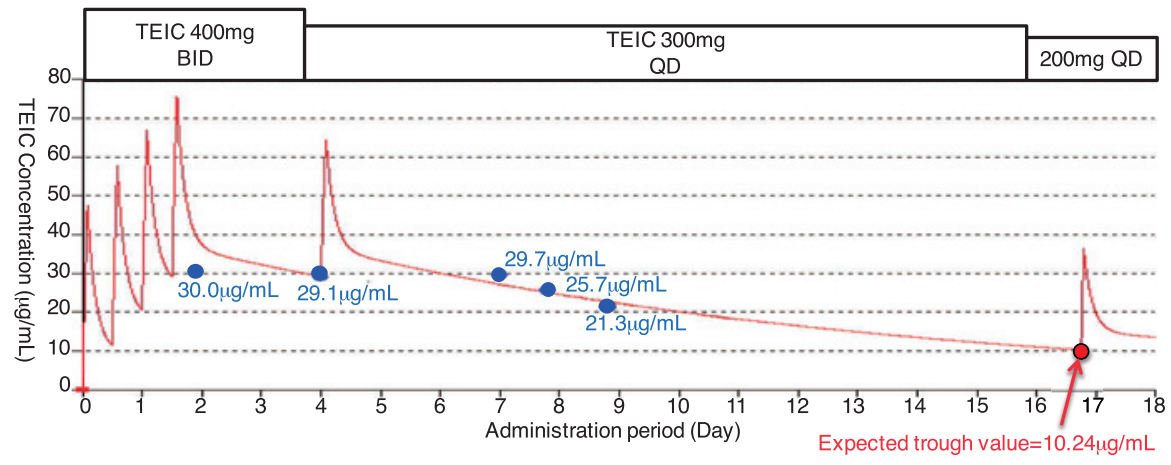

C.

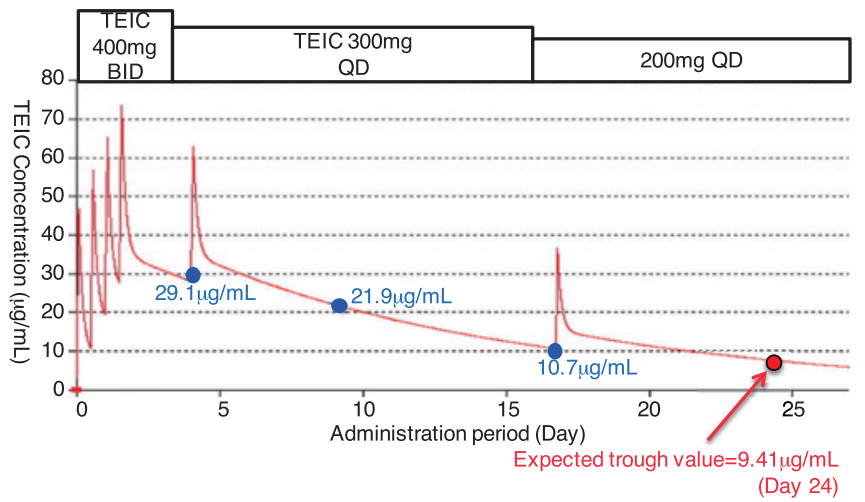

D.

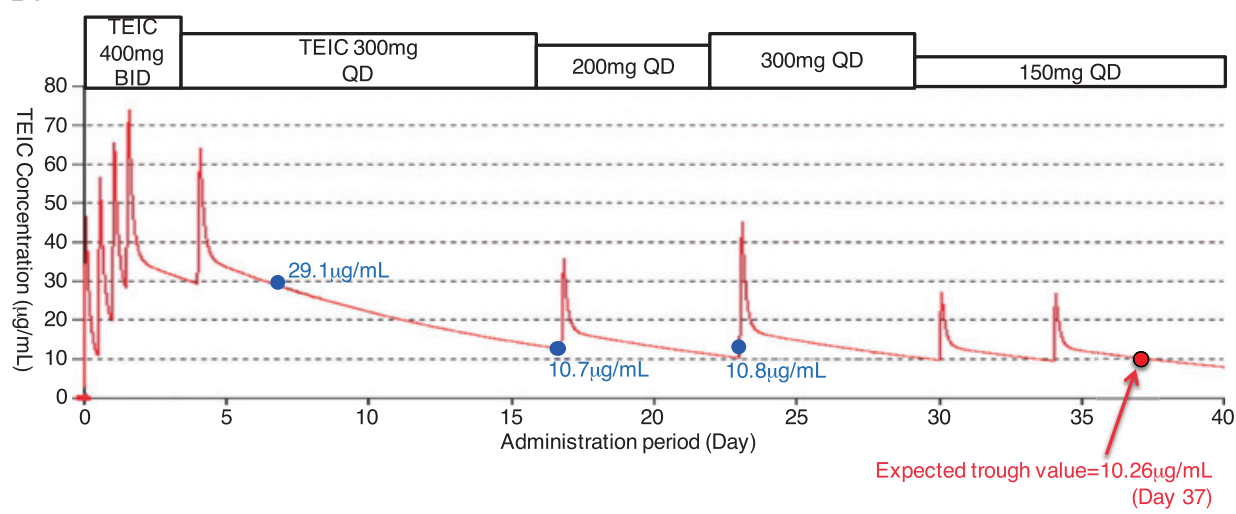

Fig. 2 We recalculated the treatment plan several times based on the absolute teicoplanin concentration and re-evaluated the patient's renal function.

A: After determining the teicoplanin blood concentration on day 4, we created a model showing administration of $300 \mathrm{mg}$ twice daily on day 11. B: After determining the teicoplanin blood concentrations on days 7, 8, and 9, we created a model showing administration of $200 \mathrm{mg}$ twice daily on day 17. C: After determining the TEIC blood concentration on day 17, we estimated the teicoplanin blood concentration on day 24. D: After determining the teicoplanin blood concentration on day 24, we developed the administration plan for the subsequent days. 
were able to eradicate MRSA from the bloodstream.

\section{Discussion}

We often encounter MRSA sepsis by translocation from sites of colonization. The virulence of MRSA does not differ much from that of methicillin-sensitive $S$. aureus. However, an MRSA infection can make a nosocomial infection more difficult to control in immunocompromised patients. Antibiotics against MRSA are of several types; therefore, the optimal antibiotic should be selected on the basis of the patient's condition and the site of infection. The TDM of glycopeptides and aminoglycosides is important to maintain the optimally effective blood concentration and to prevent toxicosis.

To treat the present case of MRSA sepsis, we selected teicoplanin. Because of the patient's history of red man syndrome, vancomycin was excluded as a candidate. Linezolid reportedly increases the risk of thrombocytopenia in patients with renal failure. Therefore, because the platelet count in this patient was always extremely low (approximately 10,000/ $\mu \mathrm{L}$ ), linezolid was also excluded as a candidate. Daptomycin is a lipopeptide antibiotic, whereas vancomycin is a polypeptide antibiotic. We avoid using daptomycin when preparing for vancomycin-resistant pathogens. Aminoglycosides administered for a long time to patients undergoing peritoneal dialysis have been associated with a high rate of ototoxicity $^{3}$.

Teicoplanin has less renal and skin toxicity than vancomycin, and we are able to analyze teicoplanin blood concentrations in our hospital. However, teicoplanin is a novel glycopeptide with considerable pharmacokinetic differences from vancomycin in terms of its long elimination half-life and tricompartmental distribution after intravenous administration ${ }^{6}$. Because teicoplanin is excreted mainly in urine, renal failure is associated with a prolonged half-life and accumulation in the body ${ }^{7}$. In addition, no standard regimen has been available for treating patients with renal failure who are undergoing continuous ambulatory peritoneal dialysis (CAPD).

We decided to devise a treatment plan that considered the dose and dosing interval. To predict the dose and dosing interval, patient factors (e.g., liver disease, kidney disease, hemodialysis, obesity, age, and pharmacokinetics) and drug factors (administration method, dosage form, and monotherapy or combination therapy) are important. Although teicoplanin administered at $10 \mathrm{mg} / \mathrm{kg}$ every 24 hours has been proposed for patients undergoing $\mathrm{CAPD}^{8}$, the only pharmacokinetic studies available were of single-dose teicoplanin in patients undergoing CAPD'. No reports have discussed the pharmacokinetics of multiple doses of teicoplanin administered to patients with CAPD. In addition, the pharmacokinetics of teicoplanin is significantly affected by inter-individual variation in albumin concentrations because the proteinbinding capacity of teicoplanin is higher than that of other antibiotics ${ }^{10}$.

In the present study, we used PEDA to determine the pharmacokinetics of teicoplanin in a patient undergoing CAPD. On the basis of a compartmental model, PEDA can graph the change in blood concentration with the dose, blood concentration, distribution volume, and elimination rate constant. Additionally, we used Bayesian estimation, with the blood collection time and blood concentration for more than 1 sample, and predicted the corrected blood concentration; thus, more accurate treatment planning was possible. Using this program, we calculated the teicoplanin pharmacokinetics, established an optimal dosage regimen, and monitored teicoplanin concentrations.

In conclusion, drug administration with TDM, using the PEDA program, might be a safe and effective therapeutic approach for patients with severe complications, such as renal failure in congenital immune deficiency.

Conflict of Interest: The authors have no conflicts of interest to disclose.

\section{References}

1. Ochs HD: The Wiskott-Aldrich syndrome. Semin Hematol 1998; 35: 332-345.

2. Liu $\mathrm{CH}, \mathrm{Wu} \mathrm{KH}$, Lin TY, Wei CC, Lin $\mathrm{CY}$, Chen $\mathrm{XX}$, Lee WI: Wiskott-Aldrich syndrome with IgA nephropathy: a case report and literature review. Int Urol Nephrol 2013; 45: $1495-1500$

3. The POC-IT Center of The Johns Hopkins University: The Johns Hopkins POC-IT ABX Guide.

4. Japanese Society of Chemotherapy: The antibiotics TDM guideline. Nihon Kagakuryouhou Gakkai Zasshi 2012; 60: 393-445.

5. Higuchi S, Aoyama T, Horioka M: PEDA: a microcomputer program for parameter estimation and dosage adjustment in clinical practice. J Pharmacobiodyn 1987; 10: 703-718.

6. Shea KW, Cunha BA: Teicoplanin. Med Clin North Am 1995; 79: 833-844.

7. Derbyshire N, Webb DB, Roberts D, Glew D, Williams JD: Pharmacokinetics of teicoplanin in subjects with varying degrees of renal function. J Antimicrob Chemother 1989; 23: 869-876.

8. Stamatiadis D, Papaioannou MG, Giamarellos-Bourboulis EJ, Marinaki S, Giamarellou H, Stathakis CP: Pharmacokinetics of teicoplanin in patients undergoing continuous ambulatory peritoneal dialysis. Perit Dial Int 2003; 23: 
$127-131$

9. Guay DR, Awni WM, Halstenson CE, Kenny MT, Keane WF, Matzke GR: Teicoplanin pharmacokinetics in patients undergoing continuous ambulatory peritoneal dialysis after intravenous and intraperitoneal dosing. Antimicrob Agents Chemother 1989; 33: 2012-2015.

10. Yamamoto T, Yasuno N, Katada S, Hisaka A, Hanafusa N, Noiri E, Yahagi N, Fujita T, Suzuki H: Proposal of a phar- macokinetically optimized dosage regimen of antibiotics in patients receiving continuous hemodiafiltration. Antimicrob Agents Chemother 2011; 55: 5804-5812.

(Received, November 13, 2015)

(Accepted,

May 17, 2016) 